\title{
EL ESPAÑOL COLOQUIAL COMO ESTRATEGIA PEDAGÓGICA: RASGOS Y RELACIÓN CON EL GÉNERO DISCURSIVO EN EL AULA UNIVERSITARIA
}

Juan García-Cardona

\section{(c) $(1) \Theta \Theta$}

Doi: https://doi.org/10.15517/rfl.v46i2.42630

URL: https://revistas.ucr.ac.cr/index.php/filyling/index 



\title{
EL ESPAÑOL COLOQUIAL COMO ESTRATEGIA PEDAGÓGICA: RASGOS Y RELACIÓN CON EL GÉNERO DISCURSIVO EN EL AULA UNIVERSITARIA
}

\author{
COLLOQUIAL SPANISH AS A PEDAGOGICAL STRATEGY: \\ FEATURES AND RELATION WITH THE DISCURSIVE GENRE \\ IN THE UNIVERSITY CLASSROOM
}

\author{
Juan García-Cardona
}

\begin{abstract}
RESUMEN
La clase universitaria conforma una situación comunicativa que se ha caracterizado, en ocasiones, con una alta formalidad. En relación con la situación que se establece en el aula, el docente escogerá un lenguaje apropiado. Con esta investigación pretendemos analizar las características de la estrategia lingüística de varios profesores de la Universidad de Málaga mediante la identificación de rasgos propios del español coloquial, con la intención de determinar si estos rasgos predominan en el lenguaje empleado por los docentes. La coloquialidad se ha conformado como un registro informal propio de la conversación, por lo que la presencia de estos rasgos coloquiales en el aula nos permitirá aproximarnos a la situación comunicativa en la que se desarrolla una clase universitaria. La muestra consta de siete profesores de los cuales se han medido de forma cualitativa 20 variables propias del español coloquial. Ordenados de menor a mayor, los sujetos de estudio han presentado 10, 13, 14, $15,18,18$ y 19 de todos los rasgos propuestos, lo que sugiere un predominio de la coloquialidad en la clase universitaria.

Palabras clave: docente; coloquialidad; discurso académico; universidad; género discursivo.
\end{abstract}

\begin{abstract}
The university class shows a communicative situation that has been characterized, sometimes, as a high formality discourse. In relation to the situation that is established in the classroom, the teacher will choose an appropriate language. With this research, we intend to analyze the characteristics of the linguistic strategy of several professors of the University of Malaga, by identifying typical features of colloquial Spanish, with the intention of determining whether these features predominate in the language used by teachers. Colloquiality has been defined as an informal register of the conversation itself, so the presence of these colloquial features in the classroom will allow us to approach the communicative situation in which a university class develops. The sample consists of seven professors of which 20 variables characteristic of colloquial Spanish have been measured qualitatively. Ordered from least to greatest, the study subjects have presented 10, 13, 14, 15, 18, 18 and 19 of all the proposed features, suggesting a predominance of colloquiality in the university class. Keywords: teacher; colloquiality; academic discourse; university; discursive genre.
\end{abstract}

M. A. Juan García-Cardona. Estudiante de doctorado en la Universidad de California, Davis. Estados Unidos. Correo electrónico: jcardona@ucdavis.edu

Recepción: 01- 11- 19

Aceptación: 20- 01- 20 


\section{Introducción}

El enfoque pedagógico del desarrollo de una lección universitaria rara vez tiene en cuenta el papel que el lenguaje del profesor toma en todo este proceso. Con el desarrollo del presente trabajo tratamos de proponer un estudio desde una perspectiva lingüística que tendrá en cuenta la estrategia seguida por los docentes y sus rasgos característicos. Para medir el lenguaje empleado por los profesores utilizaremos como referencia el español coloquial, sobre el que estableceremos distintos grados.

En primer lugar, se revisarán las principales líneas de investigación del español coloquial; para ello, utilizaremos primordialmente la tipología establecida por Briz Gómez (1996 y 2011), la cual, además, se acotará con los estudios de otros autores. Posteriormente, describiremos la investigación que se ha realizado sobre siete profesores pertenecientes al grado de Filología Hispánica de la Universidad de Málaga.

El estudio tomará como referencia la tipología del español coloquial descrito en el marco teórico y medirá los rasgos de "coloquialidad" que el profesor en cuestión presenta. Para realizar este estudio hemos transcrito el desarrollo de una de las clases de cada uno de los profesores que han colaborado en el trabajo, ya sea mediante la grabación de esta, o a partir de anotaciones -dependiendo de la elección del profesor-. El periodo de clase es de 90 minutos, de los que se han transcrito 20 de ellos repartidos en la etapa inicial, intermedia y final de la clase para evaluar el progreso. Posteriormente, hemos situado a los sujetos de estudio en una escala de 20 rasgos dividida en tres grados: alto, medio y bajo.

Además, uno de los objetivos principales será constatar el género discursivo que predomina en el desarrollo de las clases universitarias según los datos que hemos obtenido en esta fase y que, tradicionalmente, se ha ligado a la conferencia. Sin embargo, la mayoría de los aspectos estudiados parecen pertenecer al género conversacional. Esto significaría un cambio progresivo en el paradigma académico que daría lugar a distintas vías de investigación, las cuales hemos tenido en cuenta en nuestro trabajo.

\section{El español coloquial}

A la hora de abordar el español coloquial, debemos delimitar una serie de elementos que nos permitirán caracterizar el uso del lenguaje que emplean los sujetos estudiados. En nuestra investigación, tomaremos el punto de vista de este lenguaje coloquial o informal como un registro lingüístico integrado por un conjunto de variables contextuales y sociolingüísticas que determinarán su uso.

Hudson (1982) define registro lingüístico como "variedad en el sentido más bien exiguo de conjuntos de elementos lingüísticos que poseen todos la misma distribución social, es decir, que aparecen todos bajo las mismas condiciones" (p. 60). Por tanto, la condición que parece cumplirse es el uso del registro coloquial como consecuencia de una situación comunicativa apropiada para el surgimiento de este.

La estrategia lingüística utilizada por el profesor en la impartición de sus clases dependerá de una serie de variables que tendremos en cuenta en el trabajo de campo, como el canal comunicativo. En resumen, "el español coloquial refleja un tipo de registro de habla que se emplea prototípicamente en conversaciones mantenidas en situaciones comunicativas informales" (Briz Gómez, 2011, p. 36), si bien puede ser utilizado en otros tipos de discurso 
distintos a la conversación. Las principales variables contextuales que debemos tener en cuenta para determinar el registro lingüístico utilizado son, según Briz Gómez (2011), la formalidad, la especialización y la planificación. Por tanto, podría parecer una inadecuación del registro coloquial a la situación comunicativa de una clase magistral, caracterizada por un alto grado de estos tres parámetros.

Como podemos apreciar, el desempeño de una clase universitaria se consideraría una situación formal, propia de la conferencia, que requiere un uso de vocabulario técnico especializado y que, aunque pertenece a un ámbito comunicativo oral, se encontraría en el marco de la conferencia o el discurso, en la que el emisor transmite una gran cantidad de información a un destinatario, el cual, en algunos casos, puede actuar solo como receptor. No obstante, aunque la especialización y, en cierta medida, la planificación son requeridas en el desarrollo de la clase universitaria, la formalidad dependerá de la situación comunicativa que se establezca, lo que puede producir un contexto informal que propicie este registro.

Sin embargo, un profesor puede utilizar distintos registros durante la lección con el fin de conseguir una reacción determinada en el alumnado. La utilización de ciertos rasgos propios de un registro coloquial, siempre dentro de un ámbito culto, no debe implicar un vocabulario pobre o que no se adecúe a la tecnicidad necesaria para el desarrollo de dicha asignatura. Briz Gómez (2011, p. 35) identificaba los principales errores a la hora de definir lo coloquial, como lo son la confusión de tal registro con lo vulgar, la no diferenciación entre nivel de habla y nivel de lengua y, la identificación del registro coloquial única y exclusivamente con un solo tipo de discurso: la conversación.

\subsection{Definición}

Si queremos definir con exactitud el lenguaje coloquial o informal, debemos recurrir a figuras como la del hispanista Werner Beinhauer, uno de los pioneros en el estudio del español coloquial. Según Beinhauer (1991, p. 9) “entendemos por lenguaje coloquial el habla tal como brota, natural y espontáneamente en la conversación diaria, a diferencia de las manifestaciones lingüísticas conscientemente formuladas".

Además, este registro posee una serie de medios expresivos propios formados por un amplio repertorio de elementos tales como los sintáctico-estilísticos; los léxicos, como vocablos y giros; así como medios dinámicos de entonación, gesto y mímica. Cabe decir que no solo debe atribuirse el lenguaje coloquial a lo oral, ya que también comporta una gran importancia en el lenguaje escrito.

Se atribuyen distintas características a este registro del lenguaje, tales como la familiaridad, la cotidianeidad, la identificación con un tono informal, su gran expresividad y su componente subjetivo, un léxico peculiar -no especializado- que dista de los registros estrictamente formales y la utilización de expresiones pintorescas, si bien no debemos caer en los prejuicios antes enunciados. Ya Beinhauer (1991), en la definición que aportábamos anteriormente, atribuía al español coloquial rasgos destacados como la espontaneidad, la naturalidad y la falta de planificación.

Briz Gómez (2011) resume con gran acierto los aspectos fundamentales que debemos tener en cuenta a la hora de afrontar el estudio del español coloquial: se trata de un registro que no se limita a una clase social, ni es uniforme, y que se ha asociado con una simplificación del lenguaje formal; además, no se limita al ámbito oral, puede manifestarse también en el escrito; 
y por último, el lenguaje coloquial puede aparecer en varios tipos de discurso, si bien se asocia normalmente con la conversación por ser concebida como un contexto en el que se emplea un uso más auténtico del lenguaje. Por ello, aunque los registros y contextos de uso sean aplicados normalmente a una conversación, son también aplicables a otros tipos de discurso.

Una cuestión fundamental es la diferenciación entre registro y medio. No debemos confundir lo coloquial con lo oral, un tema que también trataremos en los resultados. Algunos autores han afirmado que uno de los factores que determina el registro de un hablante puede ser el medio por el que se produce la comunicación, es decir, si es oral o escrita (Wardhaugh y Fuller, 2014). Sin embargo, no debe confundirse el estilo que un hablante escoge intencionadamente acorde a una determinada situación comunicativa frente al medio en el que se establece tal situación: no todo lo coloquial es oral ni todo lo escrito es formal. Tanto lo oral como lo escrito pueden comportar estilos más o menos formales.

\subsection{Características del español coloquial}

Entre los hechos lingüísticos verbales, extraverbales y paralingüísticos que caracterizan el lenguaje coloquial hemos incluido factores sintácticos, estrategias contextuales, morfosintácticas, fónicas, léxico-semánticas y, por último, ciertos aspectos del paralenguaje. A continuación, resumiremos brevemente cada uno de estos factores, que además serán incluidos como variables en el estudio cualitativo.

En primer lugar, en cuanto a las variables sintácticas, hemos tenido en cuenta:

- Sintaxis concatenada: alude a la acumulación de enunciados no necesariamente independientes conforme vienen a la mente del hablante (Narbona Jiménez, 1989). Se contraponen a la sintaxis incrustada, propia del registro formal y que presenta una elaboración mayor, no surgida de la espontaneidad propia de la oralidad.

- Parcelación: la acumulación de enunciados y de la concatenación provoca una sintaxis parcelada, no por ello menos trabada (Narbona Jiménez, 1989). No hay un orden específico ni el hablante se preocupa en suministrar la información de una manera planificada.

- Rodeo explicativo: tanto la sintaxis concatenada como la parcelación han favorecido la paráfrasis y los continuos rodeos (Briz Gómez, 2011). Esto tiene como consecuencia un avance lento de la información, mientras que en el mensaje planificado se intenta transmitir una información concreta de la manera más concisa posible.

- Redundancia: la conversación coloquial presenta un alto grado de redundancia en las intervenciones (Narbona Jiménez, 1989). Dentro de la redundancia, podemos distinguir entre repeticiones y reelaboraciones; ambos son mecanismos de formulación con una amplia función discursiva, a pesar de que algunos autores únicamente le atribuyen un papel retardatario.

- Unión abierta: "la unión entre los enunciados es abierta, sin fuertes ataduras sintácticas, pero no por ello menos cohesionados" (Narbona Jiménez, 1989, pp. 167-180). Este es uno de los argumentos fundamentales cuando se habla de la poca elaboración o el carácter simplista de la sintaxis coloquial, la utilización de yuxtaposición y coordinación frente a la subordinación.

- Orden pragmático: se trata de un desplazamiento informativo que puede comportar un movimiento a la izquierda (pre-temas) y colocarse al inicio de la oración, o a la derecha 
(post-temas), como una especie de "coda reconocedora de algo mencionado con anterioridad" (Briz Gómez, 2011, p. 78).

- Estilo directo: un rasgo propio de la conversación coloquial es la continua presencia de relatos. Se trata de un relato dramatizado que puede corresponderse con una anécdota de uno o más hablantes, contada desde un marco espacial, temporal y personal propio. Se trata de "un recurso vivificador y actualizador de una historia pasada, rasgo en relación estrecha con el carácter inmediato y actual de la conversación coloquial” (Briz Gómez, 2011, p. 82).

Las estrategias contextuales que se han barajado en el estudio son las siguientes:

- Elipsis: es común la omisión elementos que no necesitan volver a ser repetidos en el lenguaje coloquial. Esta se caracteriza por "la elipsis frecuente no sólo gramatical, sino contextual, y la alta referencia fórica y, sobre todo, exofórica" (Briz Gómez e Hidalgo Navarro, 1998, p. 127).

- La deixis y el tuteo: sobre la deixis se trata la cuestión de la persona gramatical en que hablan los interlocutores, siendo la principal el 'yo' pues la conversación coloquial comporta un “carácter egocéntrico” (Briz Gómez, 2011, p. 83) y, el 'tú'. Además, con respecto al tuteo, cuyo uso depende de factores como la edad o la cortesía, Hidalgo Navarro (1996/1997, p. 170) observa "una tendencia al tuteo generalizado especialmente considerable en el registro coloquial".

- Enunciados suspendidos o incompletos: estos enunciados presentan una gran diversidad tipológica. Según el autor que consideremos, podemos hablar de "enunciados incompletos" (Hidalgo Navarro, 1997, p. 28) o "enunciados suspendidos" (Moreno Herrero, 1996, p. 123). Coinciden en que son enunciados cuyo significado se ve completo por el contexto en el que se utilizan.

Las estrategias morfosintácticas incluidas en el estudio quedan enumeradas a continuación:

- Intensificadores: mediante la intensificación somos capaces de transmitir un mensaje mayor que el que realmente se dice con palabras. Arce Castillo (1999) distingue una serie de elementos lingüísticos para conseguir esta intensificación, entre los que destaca los superlativos y comparativos, los prefijos y sufijos, los cuantificadores, las formas numéricas, los enunciados exclamativos, la enumeración, los enunciados suspendidos, la repetición, las onomatopeyas y algunos otros factores que también se han tomado en cuenta.

- Atenuantes: Briz Gómez (2011) establece una serie de recursos lingüísticos que se emplean para atenuar, entre los que destacan el uso de diminutivos, cuantificadores o partículas, perífrasis, reducción u omisión de fonemas de una palabra, uso de formas temporales condicionales, la modalidad interrogativa, entre otros.

- Conectores pragmáticos: mantienen la tensión comunicativa y el hilo del discurso mediante la encadenación de las unidades del habla, lo cual permite la transición de unos temas a otros en el proceso comunicativo. Son denominados por Gili Gaya (1981, p. 326) como "enlaces extraoracionales", que "relacionan a veces la oración en que se hallan con el sentido general de lo que se viene diciendo".

Las estrategias fónicas incluyen variables como: 
- Entonación: es utilizada por el hablante para cumplir una serie de objetivos, tales como "organizar los contenidos informativos, cohesionar su mensaje y realzar, por razones subjetivas o de índole pragmático-comunicativa, algunos de los elementos" (Briz Gómez, 2011, p. 140).

- Pausa: Briz Gómez (2011) distingue entre las pausas extralingüísticas, que son aquellas producidas por factores externos a la situación comunicativa y que no son relevantes en cuanto a su sentido; y las lingüísticas, las cuales responden a una función determinada y que sí son pertinentes para comprender el mensaje.

- Alargamientos fónicos: de nuevo, Briz Gómez (2011, p. 94) distingue entre alargamientos producidos por "causas externas al propio discurso [...], su valor es el de servir de apoyo para pensar lo que se va a decir; en otras ocasiones son alargamientos significativos que actúan como refuerzos del decir y/o dicho".

Los factores léxico-semánticos considerados propios del lenguaje coloquial son:

- Frecuencia léxica: la cuestión de la frecuencia léxica ha sido estudiada por diversos autores. Briz Gómez (2011, p. 97) destaca "el uso de unidades léxicas de significado extenso, menos marcado semántica y estilísticamente", por tanto, el registro coloquial quedará directamente ligado a las palabras comodín.

- Léxico argótico: el uso de argot perteneciente a clases sociales determinadas (Rodríguez, 1996). No debemos confundirlo con la jerga, que se refiere a una estratificación desde el punto de vista profesional, frente al argot, que queda delimitado por el ámbito social. En definitiva, el argot puede definirse como el lenguaje usado por aquellos grupos sociales situados al margen de la sociedad, como es el caso de los delincuentes (Pérez Santamaría, 2001).

Por último, en el paralenguaje hemos complementado los rasgos incluidos en estudios coloquiales con la obra de Davis (1996), y se han tomado como variables:

- Código quinésico: incluye tanto los gestos simbólicos como los gestos icónicos. Los simbólicos son aquellos "cuyo significado está convencionalizado y por ello pueden actuar separados de la expresión verbal, incluso sustituirla” (Briz Gómez, 2011, p. 101). Los icónicos, por su parte, dependen del contexto, suelen acompañar al código verbal y pueden ser de carácter deíctico, espacial o temporal.

- Gestos rítmicos: se diferencian de los anteriores por no contener un significado específico, sino que se repiten de forma continuada en el discurso y se ven potenciados en el registro coloquial.

- Código proxémico: se refiere al "estudio de cómo el hombre estructura inconscientemente el micro-espacio" (Davis, 1996, p. 107), lo que engloba la distancia y el contacto entre los interlocutores y el uso del espacio entre otros.

\section{Preguntas e hipótesis}

Las preguntas que se pretenden abordar con esta investigación atañen a los rasgos lingüísticos que comportan la estrategia de un profesor, a través de un estudio de coloquialidad y su relación con la situación comunicativa que se establece en clase. Por tanto, ¿se adecúan los rasgos lingüísticos que comportan los profesores al género discursivo que se aprecia en el aula? Y a profundidad sobre la situación comunicativa, ¿se asemejan los rasgos estudiados a aquellos propios de la conversación? Partimos de las siguientes hipótesis: 
a. La primera hipótesis plantea que el lenguaje utilizado por el profesor queda determinado por la situación comunicativa que se establece en el aula. Aunque un profesor pueda planear una estrategia con un fin determinado, como la de mantener un lenguaje cercano al alumno y de fácil acceso, este quedará propiciado por las condiciones contextuales que se establezcan. Para evaluar esto, debemos determinar el contexto comunicativo y relacionarlo con el lenguaje que el profesor utiliza en el desarrollo de las clases. Tomamos como referencia la noción que explica el lenguaje coloquial como aquel que se produce en ámbitos conversacionales, o como su mismo nombre indica, el lenguaje propio del coloquio.

b. La segunda proposición afirma que ha habido un cambio de paradigma en el ámbito académico. Si los distintos manuales metapedagógicos han considerado, como bien propone Robles Garrote (2013), que la conferencia es el género académico-científico por excelencia, parece haberse producido un cambio en el modelo discursivo planteado en el desarrollo de las clases a uno conversacional que daría lugar a un tipo de lenguaje determinado. Una hipótesis que quedaría directamente relacionada con la anterior. Es decir, que las clases en la Universidad, concretamente en la de algunos profesores de la Facultad de Filosofía y Letras de Málaga, ya no son conferencias. Sería interesante determinar si el género en el que se enmarcan forma parte de uno intermedio entre la conferencia y la conversación, o más bien se corresponde con una conversación no prototípica.

\section{Metodología}

Para llevar a cabo la presente investigación hemos utilizado un informe compuesto por la tipología del español coloquial de Briz Gómez (2011), el cual hemos rellenado con la intención de determinar el grado de coloquialidad que presentan los profesores. En el transcurso del trabajo explicaremos cómo se ha establecido la escala de grados de rasgos coloquiales y cómo se puntúa en ella. A la hora de escoger a los profesores estudiados, hemos recurrido al muestreo al azar de aquellos que imparten asignaturas en el grado de Filología Hispánica de la Universidad de Málaga. No obstante, algunos docentes no estaban conformes con su colaboración en el estudio y rehusaron participar.

El estudio podía realizarse siguiendo dos procedimientos según la elección del profesor. La propuesta prioritaria era la grabación de una clase al azar, realizada por un alumno procedente de la clase que imparte el profesor en cuestión -la presencia del investigador podría condicionar el habla del profesor y tener consecuencias negativas en el resultado final del estudio-, a partir de la cual se ha realizado una transcripción de 20 minutos en total del desarrollo de la clase. De estos 20 minutos, 5 pertenecen al inicio de la clase, 10 minutos a la fase intermedia y, otros 5 minutos, al final de la clase.

Algunos profesores se han negado a la grabación de su clase con la intención de preservar su privacidad. El procedimiento alternativo consistía en el estudio a partir de notas durante el desarrollo de varias clases, también basándonos en el inicio, mitad y final de clase; pero teniendo en cuenta que no es posible recoger todo el discurso del profesor de forma manuscrita. Por ello, este procedimiento incluye oraciones enunciadas por los profesores de forma aislada, lo cual nos impide estudiar los rasgos de coloquialidad de la misma forma que con el método prioritario. Ambos métodos recogían una muestra espontánea, pues no se especificó a los profesores qué día serían estudiados. El día escogido para la recolección de muestras fue decidido de manera arbitraria. 
Una vez hemos obtenido una muestra transcrita que permita determinar los rasgos del profesor en cuestión, se ha rellenado el informe basado en la tipología que hemos expuesto en la primera parte del trabajo y que está compuesto de un total de 20 ítems, a partir de los que hemos determinado el grado de coloquialidad que presenta cada uno de los profesores. En el próximo apartado se desarrollará el funcionamiento de estos grados.

\subsection{Muestra}

La muestra de profesores incluye un total de siete sujetos, tres hombres y cuatro mujeres, repartidos en los cuatro años de grado universitario de Filología Hispánica. La investigación es totalmente anónima y no se aportará el nombre de ninguno de los profesores ni rasgos que permitan identificarlos. Por ello, a la hora de referirnos a los distintos docentes utilizaremos un código formado por los componentes que se detallan en la Tabla 1:

Tabla 1. Código de los docentes

\begin{tabular}{|c|l|}
\hline $\mathbf{F H}$ & Filología hispánica \\
\hline $\mathbf{H} / \mathbf{M}$ & Sexo \\
\hline $\mathbf{0} / \mathbf{1}$ & $0=$ estudio mediante anotaciones $1=$ estudio mediante grabación \\
\hline $\mathbf{1} / \mathbf{2} / \mathbf{3} / \mathbf{4}$ & Curso académico en el que imparte clase \\
\hline
\end{tabular}

Siguiendo lo anterior, la muestra está compuesta por los sujetos: FH-M11, FH-H11, FH-M12, FH-H12, FH-M03, FH-M14,FH-H04. A pesar de aportar estos datos, el reconocimiento de los docentes puede resultar una tarea prácticamente imposible, puesto que hay 10 profesores por curso académico -en el caso de asignaturas codirigidas- y a partir de este simple código no pueden extraerse conclusiones específicas.

De todos los sujetos estudiados, cinco accedieron a la grabación del desarrollo de una clase universitaria completa y dos escogieron el método de estudio mediante anotaciones. Además, la media aproximada de edad de los profesores se sitúa en los 50 años. La muestra inicial estaba compuesta por ocho profesores, dos procedentes de cada curso académico de los que uno pertenece al sexo masculino y el otro al femenino, con el fin de rellenar las cuotas que se muestran a continuación. Sin embargo, se ha excluido un sujeto de estudio perteneciente al género masculino debido a percances médicos durante la investigación, esto da lugar a una muestra final de siete integrantes.

\section{Resultados}

Como ya hemos dicho, mediante la transcripción de sectores del desarrollo de la clase de siete profesores, hemos cumplimentado el formulario de 20 ítems que, según Briz (2011), describen el español coloquial. A partir de estos ítems hemos establecido los tres grados de coloquialidad que muestran en la Figura 1: bajo, medio y alto.

Grado bajo: 0-6

Grado medio: 7-13

Grado alto: $14-20$

Figura 1. Grados de coloquialidad 
Los ítems incluidos en el informe se enmarcan en los distintos niveles lingüísticos: sintáctico, fónico, morfosintáctico, léxico-semántico y, además, incluyen algunos aspectos contextuales y nociones paralingüísticas. El método que hemos seguido para constatar si se cumplían las condiciones propias del español coloquial consiste en la enumeración de casos en los que ocurre dicho rasgo mediante etiquetas. Para ello, hemos analizado distintos segmentos de la transcripción realizada a los sujetos de estudio para comprobar si, efectivamente, existe un predominio de los ítems que hemos clasificado en el marco teórico. Usando como medida el total de rasgos linguiísticos etiquetados, hemos determinado aquellos rasgos que se han cumplido según su predominancia a lo largo de toda la clase. Por ejemplo: si en todas las referencias a la segunda persona se ha registrado un mayor uso de tú frente a usted, el rasgo tuteo ha sido positivo. Se ha realizado el mismo proceso con el resto de los rasgos linguiísticos.

Después de analizar cada uno de estos niveles, hemos sumado los ítems que se cumplían en el sujeto de estudio correspondiente a partir de su transcripción y hemos establecido un número en la escala que antes presentábamos. Una vez sumados estos valores, hemos obtenido los siguientes resultados en la escala de coloquialidad que antes comentábamos: 10, 13, 14, 15, 18, 18 y 19. En conclusión, todos los sujetos de estudio se sitúan entre el grado medio y grado alto de rasgos propios del español coloquial. Además, predomina el grado alto en cinco de los docentes.

En la Tabla 2 se han incluido a los profesores que participaron en el estudio, ordenados de menor a mayor coloquialidad. Se han marcado en verde los rasgos que predominan en la transcripción de estos profesores y, en rojo, los rasgos que se han encontrado en menor cantidad.

Tabla 2. Resultados del análisis de coloquialidad

\begin{tabular}{|c|c|c|c|c|c|c|c|c|}
\hline & & $\begin{array}{l}\mathrm{F} \\
\mathrm{H} \\
- \\
\mathrm{M} \\
1 \\
4\end{array}$ & $\begin{array}{l}\mathrm{F} \\
\mathrm{H} \\
- \\
\mathrm{M} \\
0 \\
3\end{array}$ & $\begin{array}{l}\mathrm{F} \\
\mathrm{H} \\
- \\
\mathrm{H} \\
1 \\
2\end{array}$ & $\begin{array}{l}\mathrm{F} \\
\mathrm{H} \\
- \\
\mathrm{H} \\
1 \\
1\end{array}$ & $\begin{array}{l}\mathrm{F} \\
\mathrm{H} \\
- \\
\mathrm{M} \\
1 \\
1\end{array}$ & $\begin{array}{l}\mathrm{F} \\
\mathrm{H} \\
- \\
\mathrm{M} \\
1 \\
2\end{array}$ & $\begin{array}{l}\mathrm{F} \\
\mathrm{H} \\
- \\
\mathrm{H} \\
0 \\
4\end{array}$ \\
\hline \multirow[t]{6}{*}{ Rasgos sintácticos } & Concatenación & & & & & & & \\
\hline & Parcelación & & & & & & & \\
\hline & Redundancia & & & & & & & \\
\hline & Unión abierta & & & & & & & \\
\hline & Orden pragmático & & & & & & & \\
\hline & Estilo directo & & & & & & & \\
\hline \multirow[t]{3}{*}{ Estrategias contextuales } & Elipsis y deixis & & & & & & & \\
\hline & Tuteo & & & & & & & \\
\hline & Enunciados suspendidos & & & & & & & \\
\hline \multirow{3}{*}{$\begin{array}{c}\text { Estrategias } \\
\text { morfosintácticas }\end{array}$} & Intensificadores & & & & & & & \\
\hline & Atenuantes & & & & & & & \\
\hline & Conectores pragmáticos & & & & & & & \\
\hline \multirow[t]{3}{*}{ Estrategias fónicas } & Entonación & & & & & & & \\
\hline & Pausa & & & & & & & \\
\hline & Alargamiento fónico & & & & & & & \\
\hline
\end{tabular}




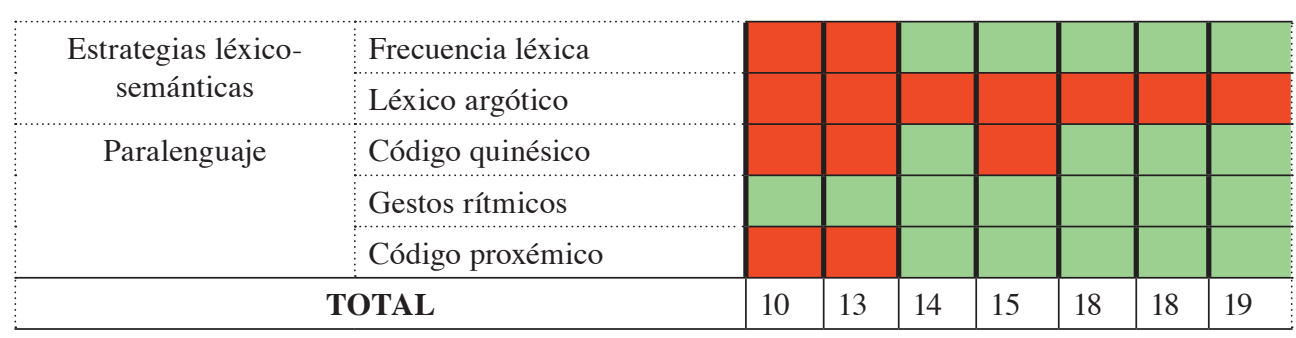

En cuanto a los rasgos sintácticos, hemos detectado todos los rasgos incluidos, menos el estilo directo, si bien parece que algunos de estos rasgos se solapan entre sí, como la concatenación y la parcelación. Es decir: el orden sintáctico propuesto por el modelo coloquial abundaba frente al orden normal establecido que puede definirse como sujeto, verbo y objeto. El estilo directo, propio del lenguaje coloquial, podría haber quedado fuera debido a la selección de 20 minutos frente a los 90 de una clase completa, al depender de la reproducción de otro interlocutor no presente y ser característico de anécdotas, algo común en la docencia. Por ello, se ha determinado una predominancia del estilo indirecto.

Dentro de las estrategias contextuales, el tuteo ha sido especialmente relevante. Este rasgo se erige como uno de los más claros para identificar una situación informal frente a una formal, por lo que aquellos profesores que utilicen el tuteo para referirse al alumno darán una sensación de mayor coloquialidad. Los profesores con menor coloquialidad presentan un uso constante de usted en los tres periodos transcritos, mientras que en uno de los casos, en el que el sujeto de estudio cumple 19 de los rasgos coloquiales, existe una vacilación en el uso de ambas formas. El primer tramo de este sujeto mantiene, de forma consistente, el uso de usted, frente al periodo intermedio y final de clase, en el que se alterna entre el tuteo y el ustedeo, si bien predomina el uso de la última forma. Resulta interesante, entonces, un estudio pormenorizado de este fenómeno pues, a pesar de ser uno de los más notables a la hora de determinar la formalidad en un contexto, no queda totalmente alineado con una mayor o menor coloquialidad. Por el contrario, los enunciados suspendidos sí quedan alineados al aparecer en aquellos sujetos más coloquiales.

La estrategia morfosintáctica incluye rasgos que se han identificado en la mayoría de los casos. La intensificación y la atenuación aparece en cinco de los siete sujetos, y también parece característico de aquellos profesores que presentan una mayoría de rasgos coloquiales. Los conectores pragmáticos, se ha identificado en todos los casos, engarzan el discurso del profesor.

En el ámbito fonético, la pausa y el alargamiento fónico son también una constante en todos los profesores, a esto se suma una entonación curva durante todos los periodos de la clase. Solo uno de los profesores mantuvo un tono monótono durante el desarrollo de la lección.

En cuanto a la estrategia léxico-semántica, aquellos profesores con menos rasgos coloquiales presentan una mayor diversidad léxica frente a aquellos con más rasgos, en los que se detecta un mayor uso de palabras comodines y generales. El léxico argótico es el único rasgo que no se ha cumplido en ninguno de los casos, puesto que incluye, como ya describimos, rasgos de habla de sectores sociales caracterizados por un nivel de instrucción bajo. Aunque se ha dado por supuesto, debemos tener en cuenta que todos los sujetos estudiados pertenecen al circuito académico universitario y que por ello forman parte de un nivel de instrucción superior. 
Por último, el paralenguaje se caracteriza por el uso de gestos, tanto simbólicos como icónicos, que conforman el código quinésico y que están presentes en aquellos profesores con más rasgos coloquiales, además de los rítmicos, que aparecen en todos los sujetos estudiados. El código proxémico, que hace referencia a la gestión del espacio físico de una clase, muestra cómo la mayoría de los profesores se movían continuamente y jugaban con la proximidad y la lejanía con respecto a los alumnos, mientras que los profesores menos coloquiales se quedaban estáticos en su mesa.

Como hemos visto, algunos rasgos aparecen en todos los docentes. La presencia de estos rasgos en todos los casos nos hace preguntarnos si se trataría más bien de rasgos de oralidad y no de coloquialidad. Las clases universitarias incluidas en el estudio son presenciales $\mathrm{y}$, por tanto, son todas orales, pero no todas comportan los mismos rasgos coloquiales. Un posible tema de futura investigación sería un estudio pormenorizado de aquellos rasgos que diferencian la coloquialidad de la oralidad.

\section{Discusión}

Si concebimos el español coloquial como una consecuencia de la conversación, es decir, el habla generada cuando se propician una serie de rasgos discursivos que categorizan el género conversacional, podríamos aventurarnos a la idea de que el desarrollo de la clase de los docentes tiene como consecuencia un alto grado de coloquialidad debido al establecimiento de un modelo de enseñanza basado en la conversación. De hecho, el término 'coloquio' es sinónimo y hace referencia a la conversación, aunque, como ya hemos dicho, este registro puede encontrarse en situaciones contextuales no enmarcadas en este género discursivo.

\subsection{Modelo de análisis conversacional}

El primer paso debe ser realizar un análisis conversacional de la situación imperante durante la lección universitaria. Es necesaria cierta matización, puesto que la conversación que pueda aparecer en el ámbito académico, y por tanto formal, distará de manera notable respecto a la que se podría establecer entre dos conocidos en un bar, como ejemplo de un contexto informal. Para el desarrollo de este análisis utilizaremos la propuesta de Gallardo Paúls (1993), en la que se aúnan las posturas de varias escuelas con bastante acierto y en la que propone una escala de rango estructural integrada por los siguientes elementos: acto < intervención (turno) $<$ intercambio (par adyacente).

En primer lugar, el acto "es la unidad mínima de la escala de rango y coincide con la unidad básica de la pragmática enunciativa"; lo relevante del acto como unidad de la conversación es su "carácter no necesariamente dialogal" (Gallardo Paúls, 1993, p. 29). Por tanto, uno de los mayores escollos que podría refutar la concepción de la lección universitaria como conversación debido a la necesidad de un intercambio bimembre, queda salvado gracias a este aspecto: incluso en aquellas clases en las que el profesor no requiere de la participación del alumnado podría establecerse un modelo conversacional.

El turno de habla, por su parte, "no es propiamente una unidad de la escala de rango, sino un hueco estructural que puede, o no, ser rellenado por una intervención" (Gallardo Paúls, 1993, p. 32). Si bien, como ya hemos visto, no es un requisito indispensable que en la conversación bimembre establecida en clase entre el profesor y el alumno se siga una 
estructura dialogal de emisión y respuesta, es algo común en gran parte de profesores permitir la intervención de alumnos, ya sea mediante una pregunta o una anotación sobre la cuestión que se esté tratando en ese momento.

Ahora bien, debemos distinguir entre dos tipos de intervenciones: aquellas en las que se aporta información y que hacen progresar la conversación, a las que llamaremos temáticas, y aquellas que se rellenan con señales de atención, continuadores y expresiones, que serán aquellos turnos de habla sin intervención real (Gallardo Paúls, 1993). Es sabido que una gran cantidad de profesores dirige la clase según la respuesta que recibe de los alumnos y, son precisamente estas señales y expresiones, como afirmar con la cabeza, lo que aporta esa información al profesor. No obstante, y como ya hemos visto, no supone una intervención del alumno.

Son mucho más aisladas y situacionales las intervenciones temáticas que dependen en muchos casos de la estrategia seguida por el profesor. Mediante la transcripción realizada de los profesores también han quedado registradas las intervenciones de los alumnos, que parecen estar relacionadas con el grado de coloquialidad que define a cada docente. Ahora bien, debemos tener en cuenta que la conclusión puede quedar sesgada, puesto que la transcripción no engloba la totalidad de la clase y se nos pueden escapar intervenciones en tramos no transcritos.

En el caso del sujeto FH-M14, que suma 10 ítems en la escala de rasgos coloquiales, solo se ha registrado una única intervención en el último tramo de la transcripción. Además, en esta intervención una alumna preguntaba la hora y comentaba si "ya nos íbamos", lo que ni siquiera podríamos considerar una intervención temática. Si nos vamos al otro extremo, el sujeto FH-H04, que cumple 19 de los 20 ítems propuestos en la tipología del español coloquial, el número de intervenciones del alumnado asciende hasta 22, todas relacionadas con el asunto que se está tratando en el desarrollo de la lección, ya sea para preguntar algún aspecto que no haya quedado claro o aportar alguna opinión al respecto.

El último elemento en la escala de rango, la noción de intercambio y el par adyacente, está caracterizada por "la productividad de sus elementos, lograda por la fusión de funciones reactivas e iniciativas en cada intervención constitutiva del par" (Gallardo Paúls, 1993, p. 39). Lo que se tiene en cuenta fundamentalmente es la colaboración de dos hablantes, por ello es considerado por algunos autores como la unidad conversacional por excelencia (Longacre, 1976; Van Dijk, 1978).

Se reproduce aquí el esquema binario o bimembre propio de la conversación, constituida por dos hablantes que, en cierto modo, intercambian información mediante las intervenciones antes explicadas. Sin embargo, estas intervenciones pueden ampliarse debido a terceros interlocutores o, incluso, intervenciones no predichas; esta es una estructura de intercambio que se considerará a partir de interacciones comunicativas de más de dos turnos. Quizá sería este factor el más difícil de reflejar en el ámbito académico. No obstante, también se establece una estructura bimembre entre el profesor, en un lado del esquema, y el alumnado, en el otro.

Estos intercambios, reflejados especialmente en aquellas clases con mayor número de intervenciones por alumno, siempre tienen en cuenta la información enunciada anteriormente y se utilizan para construir una nueva intervención sobre esta. Una propuesta beneficiosa tanto para el profesor, que puede construir sobre las dudas del alumnado, como para el alumno, que siente que tiene un papel activo en la conversación que se ha establecido y, por tanto, prestará mayor atención frente a una situación en la que es ignorado durante toda la clase, relegado a un simple papel mecanográfico de recogida de información. 
En el comienzo de esta propuesta comentábamos cómo en los manuales metapedagógicos se había considerado la conferencia como el género discursivo académico por excelencia. Y bien podríamos ver las características propias de este género en algunos sujetos de estudio de nuestra muestra. Uno de los factores principales de la conferencia es, como afirma Robles Garrote (2013), su carácter monológico. Ciapuscio (2005, p. 5) reitera esta idea manifestando que "son textos predominantemente monológicos, cuya parte final puede incluir preguntas del público, preparados para ser expuestos oralmente, en todos los casos, con apoyo visual”.

Otros autores como Camacho Adarve (2007) determinan que este turno de preguntas al final de la ponencia no pertenece al género "conferencia" en sí. Por tanto, partiendo de estos presupuestos teóricos, vemos cómo en la muestra del sujeto FH-M14, con una sola intervención y totalmente ajena al tema propuesto, podría clasificarse como una conferencia frente a otros sujetos estudiados.

En el desarrollo de una clase, la comunicación sigue siendo bilateral aunque los interlocutores alcancen un número considerablemente alto, un esquema en el que el profesor se situaría en uno de los lados y el alumno, en el otro. Rechazamos por tanto la multilateralidad que podría presentar una situación comunicativa multitudinaria puesto que los alumnos no deberían hablar entre ellos -al menos en la teoría-.

\subsection{La espontaneidad y la planificación}

Beinhauer (1991) destaca en el español coloquial los rasgos de espontaneidad y falta de planificación. Conviene ahora retomar la idea de adecuación al contexto comunicativo como aspecto determinante en el uso del español coloquial, y considerar los factores espontaneidad y planificación como fundamentales en el establecimiento de este contexto.

En el periodo primitivo de este trabajo, en el que se llevó a cabo la propuesta a los distintos profesores de cara a la participación como sujeto estudiado, también se realizó una pequeña entrevista que tenía la finalidad de recoger información sobre la estrategia que ellos mismos tenían sobre su forma de impartir materia, y así contraponerla a la ejecución real determinada por la observación del desarrollo de sus lecciones.

Uno de los factores fundamentales por los que se preguntó en esta entrevista era la planificación de las clases, un aspecto que provocó respuestas muy distintas y condicionadas por una serie de factores que a continuación desarrollaremos. La planificación de las clases era afirmativa por parte de todos los docentes estudiados, ninguno de ellos improvisaba la totalidad de sus clases, algo que resulta lógico. Hay una serie de conceptos o partes del temario que deben ser explicadas en cada una de las clases, algo que no siempre se cumple, por lo que se incluye inevitablemente un componente de planificación y organización en la muestra de todos los sujetos estudiados.

Sin embargo, otra cuestión bien distinta es la espontaneidad en el desarrollo de la lección. Muchos de estos profesores planifican el temario teniendo en cuenta ciertas desviaciones que pueden ocurrir en el transcurso de una clase, y que, por tanto, implican un componente de improvisación que dejaría de lado lo planificado. Es precisamente este factor de espontaneidad lo que propicia el uso del español coloquial.

Ahora bien, dicha espontaneidad puede aparecer por distintos factores que pudieron determinarse en el desarrollo de estas pequeñas entrevistas. Un aspecto fundamental es el dominio de la materia que se está impartiendo. Muchos profesores universitarios que aún 
no han alcanzado la titularidad, e incluso algunos que sí lo han hecho, reciben asignaturas que distan mucho de su especialidad académica. Es el caso de varios de nuestros sujetos estudiados, por lo que el desarrollo de la materia implicaría un amplio proceso de estudio por parte del docente.

Aquellos profesores que no dominen la materia o que no sientan la seguridad suficiente a la hora de impartirla, requerirán de factores externos como la lectura de citas o un ceñimiento estricto a lo planificado con anterioridad. Al no poseer los conocimientos necesarios incluidos en el plan académico de dicha asignatura, las vías improvisadas podrían limitarse debido a la insuficiencia de recursos que lo permitan. Por ello, podemos afirmar que el grado mínimo de espontaneidad en el ámbito académico podría situarse en la totalidad de una lección basada en la lectura de un artículo, lo que se consideraría como un acto de verbalización de un escrito más que un proceso de oralidad.

No debemos olvidar otros factores que no contemplaremos en este estudio, pero que poseen cierta relevancia, como la experiencia del docente y la personalidad intrínseca de cada uno los profesores. Estos factores tienen un papel muy relevante en la espontaneidad, que puede verse reducida por el miedo al fracaso o a la no aceptación por parte del alumnado, lo que puede provocar un mayor inmovilismo. Evidentemente, como ya hemos dicho, no se han incluido estos factores en la propuesta conversacional o se obviarán, por ser factores que se escapan de lo meramente lingüístico y que repercute mayormente en lo psicológico.

\section{Conclusión}

Respecto al género discursivo, podemos aproximar que aquellos profesores que presentaban una mayor cantidad de rasgos coloquiales desarrollaban la clase en una situación comunicativa mucho más cercana a la conversación que a la conferencia en lo que respecta al lenguaje. No obstante, para determinar la situación comunicativa se deben considerar muchos otros factores además del lenguaje, que en este trabajo, por cuestiones de extensión, no se han tenido en cuenta. Por ello, la conclusión que se extraiga no será exhaustiva, sino que contemplará los hechos únicamente desde el prisma del lenguaje.

Si tomamos el español coloquial como una consecuencia de la situación comunicativa y, por tanto, del establecimiento de una conversación, la posición en el grado alto de coloquialidad que presentan la mayoría de los sujetos de estudio podría estar sugiriendo un cambio en el modelo discursivo académico. Sin embargo, no debemos olvidar que el trabajo de campo de nuestra propuesta solo incluye a siete profesores del grado de Filología Hispánica de la Universidad de Málaga, un cerco muy limitado que podría implicar que muchos de los elementos estudiados puedan estar propiciados por la casualidad y que, por tanto, requieran de un estudio a una escala mucho mayor, que incluya una variedad de profesores numéricamente superior, tanto por su campo de especialización como por su procedencia geográfica.

Estos resultados arrojan nuevas cuestiones que pueden ser tratadas en futuras investigaciones. ¿Se trata la clase universitaria de una conversación prototípica? Y, en caso de que no lo sea, ¿cómo podríamos definir el género discursivo que se lleva a cabo en clase? Esta propuesta de estudio parte desde el español coloquial para determinar los rasgos lingüísticos del docente, no obstante, se hace necesario investigarlo desde otras perspectivas puesto que los resultados obtenidos no son, en ningún caso, definitivos, sino que tratan más bien de abrir nuevas vías de investigación. Confiamos en que estos resultados animen a otros investigadores 
a analizar más datos con esta y otras metodologías cuyos resultados puedan contrastarse en este estudio y llevar una visión más completa del género discursivo desarrollado en el ámbito académico universitario.

Por último, la utilización de la tipología propuesta por Briz Gómez (2011) ha sido de gran utilidad y nos ha permitido delimitar de una manera satisfactoria los rasgos coloquiales que presentan los profesores. A pesar de ello, a la hora de aplicar la tipología a la transcripción de los sujetos de estudio hemos constatado que algunos rasgos que incluye no son prototípicos del registro coloquial, y que se deben a factores situacionales, a rasgos que se corresponden con la oralidad o a al sociolecto del interlocutor.

\section{Bibliografía}

Arce Castillo, Á. (1999). Intensificadores en español coloquial. Anuario de estudios filológicos, $22,37-48$.

Beinhauer, W. (1991). El español coloquial. (F. Huarte Mortón, trad.). Madrid: Gredos.

Briz Gómez, A. (1996). El español coloquial: situación y uso. Madrid: Arco/Libros.

Briz Gómez, A. (2011). El español coloquial en la conversación: esbozo de pragmagramática. Barcelona: Ariel.

Briz Gómez, A. e Hidalgo Navarro, A. (1998). Conectores pragmáticos y estructura de la conversación. En M. A. Martín Zorraquino (Ed.), Los marcadores del discurso. Teoría y Análisis (pp. 121-142). Madrid: Arco/Libros.

Camacho Adarve, M. M. (2007). Los géneros en el discurso oral español. Espéculo: Revista de Estudios Literarios, (37), 77. Recuperado de https://biblioteca.org.ar/libros/151961.pdf

Ciapuscio, G. (2005). Esquemas calificadores modales y recursos léxico-gramaticales en la conferencia de divulgación científica. Boletín de la Academia Argentina de Letras, 70(279), 373-392.

Davis, F. (1996). La comunicación no verbal. Madrid: Alianza Editorial.

Gallardo Paúls, B. (1993). Lingüística perceptiva y conversación: secuencias. Valencia: Universidad de Valencia.

Gili Gaya, S. (1981). Curso superior de sintaxis española. Barcelona: Bibliograf, Colección Vox.

Hidalgo Navarro, A. (1996/1997). Sobre los mecanismos de impersonalización en la conversación coloquial: el tú impersonal. ELUA: Estudios de lingüística Universidad de Alicante, (11), 163-176.

Hidalgo Navarro, A. (1997). La entonación coloquial: función demarcativa y unidades de habla. Valencia: Universitat de València, Departamento de Filología Española.

Hudson, R. A. (1982). La sociolingüística. Barcelona: Anagrama.

Longacre, R. (1976). An anatomy of speech notions. Lisse: The Peter de Rider Press.

Moreno Herrero, G. (1996). La importancia del concepto de enunciado en la investigación del español coloquial: a propósito de enunciados suspendidos. Pragmática y gramática del español hablado: actas del II Simposio sobre Análisis del Discurso Oral (pp. 109126). Zaragoza: Libros Pórtico. 
Narbona Jiménez, A. (1989). Sintaxis española: nuevos y viejos enfoques. Barcelona: Ariel.

Pérez Santamaría, I. (2001). El argot y las jergas. Madrid: Liceus, Servicios de Gestión y Comunicación.

Robles Garrote, P. (2013). La conferencia como género monológico: análisis macroestructural en español e italiano. Boletín de Filología, 48(1), 127-146.

Rodríguez, B. (1997). Argot y lenguaje coloquial. Pragmática y gramática del español hablado: actas del II Simposio sobre Análisis del Discurso Oral (pp. 225-239). España: Libros Pórtico.

Van Dijk, T. (1978). La ciencia del texto. Barcelona: Paidós.

Wardhaugh, R. y M. Fuller, J. (2014). An Introduction to Sociolinguistics. Chichester, UK: Wiley-Blackwell. 